\title{
JANUS OU L'ARGENT DANS LES PRATIQUES TUTÉLAIRES
}

Richard Gaillard

Union nationale des associations familiales (UNAF) | «Recherches familiales »

2004/1 Nº1 | pages 95 à 104

ISSN 1763-718X

Article disponible en ligne à l'adresse :

http://www.cairn.info/revue-recherches-familiales-2004-1-page-95.htm

\section{Pour citer cet article :}

Richard Gaillard, "Janus ou l'argent dans les pratiques tutélaires », Recherches familiales 2004/1 ( $\left.\mathrm{N}^{\circ} 1\right)$, p. 95-104.

DOI 10.3917/rf.001.0095

Distribution électronique Cairn.info pour Union nationale des associations familiales (UNAF).

(c) Union nationale des associations familiales (UNAF). Tous droits réservés pour tous pays.

La reproduction ou représentation de cet article, notamment par photocopie, n'est autorisée que dans les limites des conditions générales d'utilisation du site ou, le cas échéant, des conditions générales de la licence souscrite par votre établissement. Toute autre reproduction ou représentation, en tout ou partie, sous quelque forme et de quelque manière que ce soit, est interdite sauf accord préalable et écrit de l'éditeur, en dehors des cas prévus par la législation en vigueur en France. Il est précisé que son stockage dans une base de données est également interdit. 


\title{
JANUS OU L'ARGENT DANS LES PRATIQUES TUTÉLAIRES
}

\author{
Richard Gaillard ${ }^{[1]}$
}

La loi $\mathrm{n}^{\circ}$ 68-5 du 3 janvier 1968 qui détermine la législation des mesures de protection juridique actuellement en vigueur s'inscrit dans le cadre de la réforme de la loi de 30 juillet 1838 sur l'aliénation. Cette dernière prévoyait déjà quelques mesures de protection patrimoniale. Lors de cette réforme de 1968, le législateur a manifesté la volonté de tenir compte des données nouvelles de la psychiatrie et du droit, mais avec le souci de répondre de façon personnalisée aux personnes auxquelles la loi s'adressait. Bien qu'elle se doive de légiférer sur la «protection des biens et de la personne », la loi de 1968 fut critiquée dès sa promulgation pour son risque de focalisation sur la protection des premiers au détriment de la deuxième.

Une ambivalence accompagne la définition de l'objet de protection des tutelles aux majeurs. Elle invite, de notre point de vue, à positionner l'argent au centre de la problématique tutélaire plutôt que dans sa périphérie. Trois constats confortent ce positionnement :

1) Les conditions et outils d'action de la protection tutélaire indiquent combien l'argent est prépondérant et occupe une place particulière dans les dispositifs. Parmi les causes légitimant une protection, l'une d'entre elles se formule ainsi : «Peut pareillement être protégé le majeur qui, par sa prodigalité, son intempérance ou son oisiveté, s'expose à tomber dans le besoin ou compromet l'exécution de ses obligations familiales. ${ }^{[2]}{ }_{\gg}$. On voit bien que ce n'est pas l'altération (physique ou psychique) qui sert de référence, mais des comportements budgétaires préjudiciables. L'utilisation de l'argent est centrale dans ces comportements stigmatisés.

[1] Les éléments présentés ici sont extraits d'une thèse de doctorat de sociologie : Richard GAILLARD, Les délégués à la tutelle. Imaginaires et réalités d'une pratique professionnelle, Université Paris 7, 2002. Les résultats de terrain qui seront présentés sont les produits de trois types de recueils de données : des entretiens individuels et collectifs avec des acteurs des tutelles (délégués, juges, tuteurs...) ; des observations de pratiques (rencontres avec des majeurs, réunions...) et des analyses de documents institutionnels.

[2] Loi n ${ }^{\circ}$ 68-5 du 3 janvier 1968, art. 1, Journal Officiel du 4 janvier 1968 en vigueur le $1^{\text {er }}$ novembre 1968 et loi n ${ }^{\circ}$ 74-631 du 5 juillet 1974, art. 1, Journal Officiel du 7 juillet 1974. Cette cause est questionnée dans les projets actuels de réforme. 
2) Le contrôle de l'argent est l'un des principaux moyens mis en œuvre dans la protection juridique des majeurs. Ceci se traduit tant par les types de droits supprimés que par les variations possibles à l'égard de ces droits. Une tutelle peut être demandée pour un majeur afin d'assurer la gestion de tout ou partie de ses biens ou revenus. Le contrôle du budget, l'incapacité juridique de la personne d'user de son argent et l'ouverture par le tuteur d'un compte autonome ${ }^{[3]}$ concrétisent la protection. Ces moyens sont mis à disposition du tuteur par l'intermédiaire de son mandat judiciaire de protection.

3) S'il est un moyen d'action privilégié pour le tuteur, le contrôle de l'argent doit aussi s'envisager comme un outil pour le juge ou le conseil de famille. L'argent dans la protection des majeurs n'importe pas seulement dans les rapports avec les personnes suivies. Il constitue un niveau d'évaluation de l'action du curateur, ou du tuteur, lequel doit rendre compte de sa gestion chaque année au greffier en chef du tribunal d'instance, ou au juge. Pour passer des actes qui engagent le patrimoine du majeur, le tuteur, doit, en plus de l'obtention de l'aval du conseil de famille ou de celui du juge des tutelles, rendre visible et évaluable son travail sur les budgets par la reddition des comptes de gestion. Cette perspective de contrôle et de recherche de transparence prend un sens particulier, car le tuteur détient une marge de liberté et d'initiative importante dans la gestion d'un budget et d'un patrimoine. La liberté de gestion, soustraite aux personnes et transmise au tuteur, reste une liberté sinon toujours surveillée, au moins exigeant un minimum de comptes rendus possibles auprès des magistrats. Partant de ces constats, une étude sur l'argent dans les pratiques tutélaires nous semble intéressante. L'argent dépasse le cadre strict du registre comptable. Lui donner une définition n'est pas simple; il faut le penser comme objet « mouvant». C'est à partir de l'enjeu des échanges sociaux, puis de la dynamique des désirs psychiques, que nous proposons d'étudier l'objet « argent ». Ces deux axes d'analyse se révèleront heuristiques pour penser, par la suite, l'argent dans les pratiques tutélaires.

\section{$\$ L'argent, l'échange, la valeur ou la réalité de leurs enjeux dans la protection tutélaire}

L'argent, l'échange et la valeur sont indissociables. Une présentation du paradigme de l'échange et une clarification des propriétés de l'argent permettent de distinguer ces notions, mais aussi de souligner les liens argent-valeur-échange et ainsi envisager leurs effets potentiels dans les pratiques tutélaires.

Si l'on entend par «échange » l'ensemble des relations par lesquelles nous cédons quelque chose (objets, services, affects... : don envers l'autre) dans l'attente ou l'espoir d'obtenir quelque chose en retour (objets, services, affects... : dette de l'autre à notre égard), l'échange avec autrui marque notre quotidien. Tout échange constitue une ronde de dons, de dettes et de contre-dons dans laquelle chacun d'entre nous occupe, alternativement, la place d'endetté et de donateur. Cette quotidienneté ne doit pas faire perdre de vue que ce type de relation est vital. L'échange peut être ainsi défini comme une «sorte de rapport social élémentaire $»^{[4]}$. Quelle qu'en soit sa forme, il constitue un paramètre

[3] Compte sur lequel sont versés les revenus et capitaux ou épargne, et avec lequel sont réglées les dépenses

[4] Henri MENDRAS, «Échanges, clientèles, organisation ? », in Éléments de sociologie, Paris, Armand Colin, 1967, pp. 175188. 
structurant pour toutes les sociétés humaines, primitives ou industrielles. Plus qu'un rapport parmi d'autres, il tient une place essentielle dans la constitution des sociétés et les relations entre individus. Ainsi, Georg Simmel dans Philosophie de l'argent, considère l'échange comme le rapport fondamental entre les individus dans la vie sociale. Défini comme ciment des sociétés, il s'impose en tant que finalité des relations sociales. Il est ce qui crée du lien entre les individus, les capte, les fait appartenir à un groupe. Il apparaît dès qu'il y a relation. L'échange n'est pas une simple action, mais une finalité avec une dimension socialisante.

L'échange, cette relation qui permet une existence sociale par la reconnaissance qu'elle entraîne, dépend cependant de la valeur. La « valeur » est une notion difficile dont il faut souligner qu'elle résulte tout à la fois d'une distance et de sa mesure. Ainsi, la valeur peut être entendue comme le résultat d'un désir, ou d'un besoin de quelque chose séparé de son contenu effectif. Elle relève d'une distanciation entre un individu désirant et une chose désirée. Cette distanciation est, de surcroît, consciente, quantifiée et mesurée. Par sa mesure, la distance donne alors naissance à la valeur. Cependant, la valeur, produit de transferts, est, par essence, subjective et relative. « À travers les preuves de la valeur, on n'appréhende jamais que le transfert d'une valeur établie à de nouveaux objets et, en aucun cas, l'essence même de la valeur, ni la raison pour laquelle, à l'origine, elle fut attachée à tel objet qui, ensuite, la répercutera sur d'autres. $\gg^{[5]}$ De cette relativité et subjectivité de la valeur, une discorde entre les protagonistes est possible et un danger peut ainsi apparaître pour l'échange. Cette discorde représente un risque car elle met en jeu la possibilité de l'échange.

Comment est-elle résolue ? Notamment par l'intermédiaire de l'argent. La réponse qu'il fournit s'appuie sur la quête d'objectivité de la valeur qui, pour se manifester, a besoin d'être traduite en symboles. La place considérable occupée par l'argent dans cette quête de symbolisation est due non à sa matière, mais à sa fonction d'objectivation. L'argent matière - n'a ainsi que peu d'importance face à cette fonction. Son histoire montre ainsi comment il a remplacé le papier, le cuivre, jusqu'au moment où sa substance fut moins primordiale que sa fonction. L'argent est donc représentant de la valeur, parce qu'il objective, et de ce fait universalise, la possibilité d'échange et la valeur.

Les propriétés fiduciaires et consensuelles de l'argent doivent également être soulignées pour comprendre la place qui est la sienne dans les échanges sociaux. L'argent n'aurait point de crédit s'il n'était inscrit dans un univers de confiance où, durant le temps de l'échange, le doute sur la valeur est suspendu. Médiatrice entre l'argent et le temps, l'interruption d'une incertitude semble être une condition nécessaire à l'utilisation de l'argent. « La fonction d'anticipation du signe monétaire est inscrite dans ce gage de la promesse de paiement qui met le doute en suspension ». Conventionnel et arbitraire, disait déjà Aristote, l'argent s'inscrit dans une relation fiduciaire, où il est un " signe d'échange, ancré dans les mécanismes de la confiance et chargé d'une valeur consensuelle $»^{[6]}$. Il évolue « sur un territoire où souffle l'esprit de la croyance, du crédit $»^{[7]}$ dans un monde de contrat, de convention tacite. Il n'est pas anodin qu'étymologiquement « créance » et « croyance » appartiennent à la même famille, car l'argent est, dans sa genèse, fiduciaire et conventionnel.

[5] Georg SIMMEL, Philosophie de l'argent, Paris, PUF, 1987, p. 21.

[6] Ibid.

[7) Jean Marie THIVEAUD, «Argent et matière. L'institution financière et l'invention de la monnaie », L'inactuel, $\mathrm{n}^{\circ}$ 5, 1996, p. 170 . 


\section{\ Le contrôle des budgets pour « maintenir le lien social»}

Sur la base de ce développement théorique, on peut émettre l'hypothèse que la suprématie de l'argent dans les échanges (associée à son pouvoir sur la possibilité d'exister socialement) et la relation fiduciaire qui les sous-tendent (découlant de la nécessaire suspension du doute sur la valeur), risquent d'agir sur la réalité tutélaire selon une perspective de contraintes, d'exclusion et de stigmatisation des personnes sous protection juridique, plus que d'une protection pour des individus fragiles.

L'absence de manipulation d'argent rime sinon avec l'absence de possibilité d'échanger, au moins avec sa réduction. Priver un individu de toute manipulation d'argent, comme dans la tutelle aux personnes majeures, revient à lui ôter un élément structurant de sa place dans la société, et à nier une forme de son existence. Diminuer les possibilités d'échanger, c'est aussi réduire les capacités de l'individu à être reconnu, et à participer à un réseau social. Que cette privation se légitime par la protection de la personne ${ }^{[8]}$, l'importance des processus sur lesquels elle porte peut entraîner les effets inverses d'une protection, à savoir, sa négation en tant que sujet social.

Au-delà de ce paradoxe, la question de la confiance risque de fragiliser plus encore la clarté des objectifs de protection. L'utilisation de l'argent semble nécessiter la suspension d'un doute au cours de la relation d'échange et sur la valeur de ce qui peut s'échanger. Cette suspension est conséquente, car un soupçon risque de remettre en cause l'échange. Partant de cet enjeu, les pratiques tutélaires apparaissent comme un moyen de stigmatiser et d'exclure des processus d'échanges des individus suspects quant à la confiance qu'il est possible de leur accorder. En effet, les causes de mises sous tutelles de personnes s'étayent sur le constat de comportements ou de traits de personnalité qui entraînent une perte de confiance. Les altérités, physique ou mentale, et la prodigalité, l'intempérance et autres dépenses inconsidérées sont préjudiciables pour la personne, mais aussi pour la confiance qui lui est faite. Or, cette perte de confiance équivaut à un danger pour l'échange entre cette personne et les autres.

Nous émettons une nouvelle l'hypothèse, celle de l'existence d'un lien entre, d'une part, le paradoxe des objectifs de protection (et les effets des moyens pour les mettre en œuvre) et, d'autre part, l'enjeu autour de la question de la confiance. En effet, parce que les échanges sont des rapports fondamentaux pour la construction individuelle et sociale, parce que l'argent est le moyen privilégié des échanges modernes et enfin parce que néanmoins, l'argent et l'échange peuvent souffrir de soupçons sur la valeur, les pratiques tutélaires s'avèrent implicitement des instances de contrôle social masquées derrière des objectifs de protection de personnes fragiles.

L'analyse de discours des délégués que nous avons interviewés, outre l'analyse des objectifs officiels et officieux repérables dans les documents institutionnels, permet de valider l'idée que les échanges, lorsqu'ils sont malgré tout possibles dans les pratiques tutélaires, tendent vers des espaces où la reconnaissance sociale est absente.

Les règles et interdictions institutionnelles contenues dans la charte de l'UNAF, par exemple, quant aux échanges entre personnes " protégées » et délégués peuvent confirmer les risques d'échanges inéquitables et stigmatisants. Cette charte insiste sur un point : les UDAF s'engagent à "veiller à ce que leurs administrateurs, représentants et salariés, n'acceptent

[8] Le contrôle du budget permettant ainsi d'éviter la dilapidation du patrimoine, et protège les possibilités ultérieures de l'individu à participer à d'autres échanges. 
aucun dons, legs, avantages, ni se livrent à aucune transaction ayant rapport avec une mesure confiée $»^{[9]}$. L'effet de l'interdit se situe sur le plan de l'échange et peut signifier un effet coercitif des tutelles. S'engager à ne pas accepter « des dons, legs, avantages, ni aucune transaction ayant rapport avec une mesure », vient, dans la logique des processus d'échange, interdire au délégué l'éventualité de recevoir quelque chose de la part des personnes qu'il suit. Si cet engagement peut être le gage d'une honnêteté des professionnels ${ }^{[10]}$, il signifie aussi l'impossibilité pour les personnes suivies de s'inscrire dans des rapports d'échange. Cet engagement les maintient à la place de celui qui reçoit. Par ce statut de bénéficiaire, et ce seul statut, elles peuvent être dans la position paradoxale d'être endettées, car dans l'impossibilité de rendre en échange de ce bénéfice et de cette protection.

Cet interdit équivaut ainsi à une forme de surendettement symbolique du «protégé », qui ne peut être que «le bénéficiaire » d'une protection qu'il n'a, la plupart du temps, pas demandée, et contre laquelle il ne peut rien rendre en retour. Si le fait de participer financièrement au coût de la mesure (comme le précise la loi de 1968 et les écrits de service) peut être cette contrepartie, elle a, du point de vue des dispositifs, l'effet inverse. Cette participation, en étant moindre que l'estimation réelle du coût des mesures, peut signifier que l'objectif n'est pas de réaliser un échange équitable, mais de maintenir " protégé » à une place d'endetté, à des fins coercitives.

Rappelons, en effet, que cette obligation de rendre, lorsqu' on est, ou se sent « en dette », participe de « l'ordre du monde ». Dans un rapport de don et de contre-don, entre protecteur et protégé, si le second temps est impossible, il fixe le «bénéficiaire » dans un rapport de dette et met celui qui y est soumis à la merci de l'autre, lequel, par son don, (la protection), a pouvoir sur lui. Au final, l'impossibilité pour les personnes protégées d'instaurer tout autre type d'échange avec les délégués, les place dans l'impossibilité d'être dans un rapport d'échange.

Il n'est pas anodin que les personnes suivies fassent « des cadeaux, remercient, invitent à dîner $»^{[11]}$. Si le premier côté de l'échange, (celui du donateur) est inaccessible du fait de la dépossession d'argent, ils ne peuvent, même dans leur faible moyen ou sur un registre non monétaire, contre-donner et par là même être reconnus dans ce rapport d'échange.

Les délégués, recevant un salaire, peuvent aussi s'envisager en difficulté, voire « endettés », dans les rapports d'échange : les personnes suivies sont dans l'obligation d'échanger et les dispositifs les en empêchent ; les délégués, quant à eux, doivent aménager le fait de recevoir plus (un salaire) que ce qu'ils ne donnent. Les objectifs qu'ils expriment, et la place qu'ils donnent à l'argent en sont une confirmation. Ils indiquent la difficulté d'une relation d'échange déséquilibrée. Ils semblent se sentir en dette vis-à-vis des personnes suivies lesquelles, en leur laissant leur argent, et en se soumettant à leur contrôle, leur permettent de gagner, de l'argent pour vivre. Une traduction de cette situation est exprimée de deux manières :

- par le sens que les professionnels donnent à leur pratique, à savoir une volonté d'aide et non de contrôle ${ }^{[12]}$,

[9] UNAF, CIFOT, Voyage au long de la tutelle, 64 p., 1996.

[10] Il annule la possibilité d'abus de pouvoirs ou d'utilisation des capitaux des personnes de façon détournée.

[11] Les délégués rencontrés ont de nombreuses fois témoigné de ces cadeaux, invitations dont ils sont les destinataires. Si tous les délégués ne sont pas systématiquement envisagés comme éventuels héritiers (certains d'entre eux ont déclaré avoir été confronté à cette proposition), les remerciements de toutes sortes relèvent de notre point de vue de la même question : l'équité de l'échange via le contre-don et donc l'annulation de la dette.

[12] Les objectifs poursuivis peuvent alors être très éloignés des objectifs officiels : certains délégués veulent soigner les personnes, alors que cet objectif ne relève pas de leur mandat, ni de leur compétence. 
- par leur insistance quant à la non-appropriation de l'argent des personnes ${ }^{[13]}$ et la volonté de voir dans l'argent un simple moyen et non une finalité ${ }^{[14]}$.

Outre les aspects philosophiques et sociaux que nous venons de voir, l'objet argent a aussi à voir avec le domaine de la psyché. Le rapport de l'individu à ses désirs contient une incidence sur la manipulation qu'il peut avoir de son budget.

\section{$\checkmark$ Argent et désirs ou la question de la toute puissance des déégunés}

La place et les significations que prend l'argent vont dépendre de la problématique psychique de la personne et de ses singularités. Ainsi, le temps, la jouissance et le rapport substitutif de l'argent à l'égard de cette dernière, posent ce moyen d'échange moderne dans une problématique des désirs. Ces enjeux liés à l'argent ont été largement étudiés ${ }^{[15]}$.

La psychanalyse « enseigne depuis les premières intuitions et conceptualisations freudiennes, que l'argent est, pour l'inconscient de tout sujet, situé dans un rapport d'équivalence symbolique avec les notions d'excrément, de cadeau, de pénis et d'enfant $»^{[16]}$. Sandor Ferenczi, en écho aux travaux de Sigmund Freud, comprend l'argent et l'intérêt à son égard comme le bout d'une chaîne symbolique permettant de gérer la jouissance anale. «La jouissance, liée au contenu intestinal, devient plaisir (acceptable par le surmoi) procuré par l'argent, qui n'est rien d'autre que des excréments désodorisés, déshydratés et devenus brillants. $\gg^{[17]}$ L'argent semble donc s'inscrire dans une chaîne symbolique où il devient une forme d'ersatz, permettant la gestion de la jouissance anale.

Eugène Enriquez insiste sur les rapports particuliers qui peuvent s'instaurer entre l'argent et les désirs. L'homme, être manquant ou chercheur insatiable de plus de jouissance, est pris dans une quête de reconnaissance à l'égard d'autrui, dont la toile de fond est une problématique narcissique ${ }^{[18]}$. Représentant la puissance phallique, l'argent devient alors un moyen « d'emprise sur les autres ». Mais, promu au rang de divinité et de fétiche, dans un reversement où il devient aimé, « cet argent qui avait tué le désir, le fait renaître de ses cendres $»^{[19]}$. La situation est une situation de dupes. L'argent permet, certes, de plus en plus de satisfaction à celui qui le possède, et « il engendre le désir de nouvelles satisfactions $»^{[20]}$, mais la jouissance recherchée et illusoirement obtenue par son biais reste à jamais inaccessible.

Selon Ilina Reiss-Schimel, l'utilisation de l'argent nécessite une maturation psychique qui permette « de pouvoir séparer l'acte de vente et l'acte d'achat, ce qui n'existe ni dans le vol ni dans le troc. Cette opération suppose l'assimilation de la notion de délai dans le

[13] Sorte de discours récurrent, tous les délégués rencontrés insistent sur le fait qu'ils rappellent quotidiennement que l'argent appartient aux personnes protégées et non aux professionnels ou aux structures tutélaires.

[14] Dans tous les entretiens et pour tous les délégués, l'argent est un moyen et non une fin.

[15] Outre les auteurs cités ensuite, voir sur le sujet Pierre MARTIN, Argent et psychanalyse, Paris, Navarin Éditeur, 1984.

[16] Arnaud GILLES, «Quelques considérations sur la fonction symbolique de l'argent pour la psychanalyse. Pratiques sociales de l'argent », Revue Internationale de Psychosociologie, $\mathrm{n}^{\circ} 13,1999$, pp. 37-49.

[17] Sandor FERENCZI, Psychanalyse, œuvres complètes 2, Paris, Payot, 456 p., 1978 (éd. or. : 1913).

[18] Eugène ENRIQUEZ, « L'argent, fétiche sacré... », in Jean-Philippe BOUILLOUD, Véronique GUIENNE (dir.), Questions d'argent, Paris, Desclée de Brouwer, 1999, p. 55.

[19] Ibid., p. 55.

[20] Ibid., p. 56. 
fonctionnement psychique. De fait, c'est la dimension de la temporalité dans son ensemble qu'il faut avoir intégrée, car il est nécessaire de savoir inscrire ses désirs dans le temps sous forme de projet $»^{[21]}$. Elle insiste ainsi sur le fait que l'argent est un moyen de mettre en œuvre une réalisation de désir, mais au prix d'une capacité à la «temporaliser ».

En outre, cette temporalité est, selon Jacquelin Barus-Michel, à réintroduire dans l'histoire de l'individu. L'argent « prend peu à peu place dans l'histoire du sujet comme représentant (signification plus chargée affectivement et en partie inconsciemment) des désirs et des angoisses de l'individu engendrés dans un rapport à des objets fantasmatiques induits par les relations parentales précoces et les satisfactions, trouvées ou pas, au besoin d'amour, de sécurité et de plaisir » ${ }^{[22]}$. Tout au long de l'histoire du sujet, les significations de l'argent vont se suivre, objet de substitution fantasmatique et objet mythique du désir, il est le creuset de la jouissance. Objet polyvalent, car non réductible aux enjeux du stade anal, «il engage le corps intime ${ }^{[23]}$. Les significations de l'argent et les conduites à son égard sont alors multiples, puisqu'il peut être un objet polyvalent pour l'inconscient. Conduites idéalisantes, rationnelles, fétichistes, car « le sujet entretient avec lui des relations érotiques, ludiques et narcissiques d'identification (exhibition), de surcompensation (dépenses pour des "folies", délinquance machiste), enfin des conduites irrationnelles, aberrantes ou pathologiques (prodigalité, dilapidations, pertes compulsives), qui manifestent un rapport inflationniste à l'argent dont la valeur est manifestement autant affective qu'économique ${ }^{[24]}$. Il y a aussi, derrière ces dernières conduites, sacrificielles, de «folle générosité », la marque du sacré, de la profanation et de la culpabilité. Pour celui qui manipule l'argent inconsidérément, le risque est grand, car l'argent signifie l'engagement social, le symbolique, mais renvoie aussi à des valeurs premières. Ainsi, « son maniement pervers ou même inconsidéré fait du fauteur un damné, un réprouvé, un corrompu ; littéralement, c'est l'être qui est corrompu ».

\section{$\$ Argent et désir des déléegués et des personnes suivies}

La place de l'argent dans la pratique tutélaire s'avère ainsi chargée d'enjeux psychiques inconscients confrontés à une logique de contrôle. Non seulement le contrôle du budget d'un individu signifie le contrôle d'un espace de réalisation des désirs inconscients, mais en plus, l'individu en charge de ce contrôle se voit muni d'un pouvoir aux résonances potentiellement inquiétantes.

Les tuteurs sont en effet à une place peu confortable : celle d'être à la fois coupés d'une possible analyse des dimensions inconscientes tout en étant confrontés à un objet sous le signe de l'imaginaire échappant totalement à leur niveau d'action. Le pouvoir sur les budgets accentue les enjeux psychiques, car il leur permet une emprise considérable sur des personnes risquant alors de devenir l'objet de leur propre jouissance.

Plusieurs données de terrain confirment la réalité d'une telle situation.

[21] Ilina REISS-SCHIMEL, «Être et avoir - Stades d'évolution psychique. Pratiques sociales de l'argent », Revue Internationale de Psychosociologie, $\mathrm{n}^{\circ} 13,1999$, pp. 25-35.

[22] Jacqueline BARUS-MICHEL, “ L'argent ou la magie de l'imaginaire ”, in Jean-Philippe BOUILLOUD, Véronique GUIENNE (dir.), Questions d'argent, Paris, Desclée de Brouwer, 1999, p. 67.

[23] Ibid., p. 70.

[24] Ibid., p. 71. 
Tout d'abord, étant donnés l'existence de liens faibles entre les tuteurs et les médecins ${ }^{[25]}$, l'absence de connaissance en psychologie de la part des tuteurs et l'interdiction d'y avoir accès $^{[26]}$, les significations psychanalytiques de l'argent leur échappent. En considérant, par ailleurs, que certaines personnes sous tutelle relèvent de la psychose, forme de psychopathologie qui se structure sur une problématique de jouissance, les exigences de temporalité du désir dans l'utilisation d'une forme monétaire abstraite qu'est l'argent posent un problème, sans que les délégués ne puissent le comprendre parfaitement et travailler avec. Ensuite, la marge de liberté dans le travail sur le budget et l'absence d'instance de contrôle, autre que budgétaire, dans l'organisation des dispositifs tutélaires, ne peuvent qu'inquiéter sur le pouvoir des délégués acquis par la maîtrise totale du budget. Rien n'est garant du fait que les délégués ne peuvent être pris dans des rapports d'instrumentalisation, volontaire ou involontaire, des personnes.

Si les pratiques tutélaires peuvent s'avérer problématiques et équivoques parce qu'elles entraînent la privation d'un objet aux résonances psychiques et sociales fortes, elles le sont aussi par la situation dans laquelle elles mettent le délégué. Toute personne faisant office de tuteur est installée dans un rapport potentiellement illusoire de toute-puissance face aux désirs : les siens et ceux de la personne suivie. Ce rapport, par la culpabilité qu'il peut entraîner ou la violence qui peut en émerger, peut être la source de souffrance pour l'une comme pour l'autre. Les propos récurrents des délégués confirmant cette situation sont de trois ordres : les difficultés rencontrées, les significations attachées à l'argent, et leurs motivations pour être délégué à la tutelle.

Ainsi, au cours des entretiens, une grande partie des délégués s'est exprimée sur ses difficultés à assumer le mandat tutélaire et "le pouvoir de gérer l'argent à la place des gens ». Une déléguée dit, par exemple, son embarras à refuser ou non de l'argent aux personnes et s'interroge sur les fondements de ses exigences, "mon travail, finalement, c'est le pouvoir de dire oui ou non sur des suppléments, et d'exiger que les demandes ne reviennent pas trop souvent. Je suis mal à l'aise avec ce pouvoir, je ne sais pas pourquoi je pose ces exigences, c'est une déformation professionnelle, personnelle... ». Un autre a le sentiment d'être un «magicien » qui a «tout pouvoir, ou un apprenti sorcier qui n'a pas de limites dans les possibilités que lui procure le fait d'intervenir sur l'argent des personnes sous tutelle».

Ces propos sont intéressants pour les deux types de rapports à l'argent qu'ils indiquent et la signification qu'ils peuvent avoir en termes de jouissance. La subjectivité évoquée tout d'abord est apparue de nombreuses fois dans les discours comme partie liée au travail de délégué. Qu'elle pose problème, ou qu'elle soit acceptée, elle semble un état de fait. La fascination du pouvoir que fournit l'argent, un «outil fantastique » est, elle aussi, à prendre en compte. Ces deux manières de parler de l'argent dans les pratiques peuvent être le premier signe de la mise en jeu du fantasme de cette toute puissance. La dialectique qui en découle peut être le signe d'une dénégation de la jouissance.

Si ce niveau d'analyse s'étaye sur le registre de l'inconscient et nécessite la prudence, la culpabilité liée à l'utilisation de l'argent et la fascination-répulsion simultanée ${ }^{[27]}$ n'est pas sans lien avec les processus inhérents aux tentatives de réalisation des désirs. Ces processus s'étayant sur la culpabilité et le refoulement dans les potentielles réalisations de désirs.

[25] Sur ce point, voir : Richard GAILLARD, « Pratiques de tutelle et pratiques de soin : ambiguïtés du rapport à l'argent », Bulletin de psychologie, publication prévue en janvier 2004.

[26] Nous faisons ici référence à l'indépendance instituée par la loi de 1968 entre la protection et le soin.

[27] Il est considéré, par les délégués, comme un moyen mais, aussi, comme un mauvais objet. 
La culpabilité peut aussi se traduire par la nécessité, de la part des délégués, d'expliquer leur motivation pour les pratiques tutélaires par un effet de hasard, de circonstance, pour des raisons économiques, ou de façon plus générale, par une volonté d'aider l'autre. Cette bienfaisance constitue une réponse à la culpabilité d'avoir ce pouvoir et ce désir de toute-puissance. Il n'est pas anodin, d'ailleurs, sur ce registre de la culpabilité, que lorsque les délégués avouent des difficultés dans la gestion des budgets, ces difficultés soient notamment issues de choix à faire quand les coûts sont équivalents ou quand les décisions touchent au corps et à l'intime des personnes suivies. Les coûts équivalents révèlent leur subjectivité, " on a nos valeurs, notre éducation et on est dans une position de pouvoir ». Dans ces cas d'équivalence des coûts, est en jeu la révélation du désir des délégués, habituellement masqué par «les budgets équilibrés », considéré simultanément comme finalité et comme moyen. Ce type de difficultés met à jour ce qui se joue au nom de l'équilibre du budget.

Les difficultés, face à l'intime et au corps des personnes suivies, sont d'un autre ordre, mais peuvent également révéler cette culpabilité. Les propos suivants sont venus témoigner de cette spécificité du corps comme espace pouvant, sinon échapper au contrôle, au moins entraîner plus de souplesse. Cela «fait mal au cœur » pour certains de refuser de l'argent pour des habits. D'autres ne supportent pas qu'on l'interroge pour " l'achat d'un slip, ou des problèmes d'anus ». Or, l'argent engage le corps intime, souligne J. BarusMichel ${ }^{[28]}$, il peut parfois équivaloir à ce corps, la sexualité, et donc la jouissance.

Quand le lien se pose de manière trop directe ou trop crue, il rappelle directement la jouissance, la propre jouissance du délégué et appelle l'interdit, produit du refoulement du surmoi. Il n'est pas anodin que cette thématique corporelle, dans le discours des délégués, porte sur des zones (anales et génitales et liées à l'hygiène) importantes dans le rapport inconscient à l'argent. Même s'il ne se résume pas à cela, il faut néanmoins rappeler que le plaisir que fournit l'argent est le produit d'une sublimation de la jouissance anale qui, pour être acceptable, doit trouver un autre espace, une autre forme. C'est en cela que les aspects liés au corps et la répulsion, ou la souplesse, soulignés par les délégués, peuvent être rattachés à l'enjeu d'une jouissance qui court le risque de la culpabilité. La jouissance doit, en effet, avancer masquée pour qu'elle soit supportable, et ces zones érogènes que s'interdisent les délégués peuvent se voir comme un aménagement psychique dans la recherche de jouissance avec la manipulation de l'argent des autres. Au final, c'est la dialogique des discours et des comportements (argent outil fantastique, mais répugnant) vis-à-vis de l'argent qui nous semble être la trace d'un tel fantasme et de son refoulement nécessaire, car «surmoïquement » insupportable. Cette double-face que revêt l'argent dans les propos des délégués renvoyant aussi à la double fonction soulignée par Eugène Enriquez ${ }^{[2]}$ à l'égard des rapports entre argent et désir. L'argent, transformateur du désir, vient le canaliser, le rendre mesurable et donc le tuer, et les «budgets équilibrés » fonctionnent de cette façon pour les délégués ${ }^{[30]}$. Parallèlement, l'argent embrayeur permet de dénier la castration symbolique et donc ouvre au fantasme de toutepuissance. Il est sans limite et peut fournir aux délégués le moyen de poursuivre tous les objectifs possibles comme le soin ou leur unique et personnelle problématique narcissique. Ils courent alors le risque d'être des «magiciens » avec tous les pouvoirs.

[28] Op. cit.

[29] Op. cit.

[30] Les délégués trouvent dans la nécessité d'établir un budget équilibré une manière de répondre aux demandes d'achat quand elles sont problématiques. 


\section{$\triangleleft$ Conclusion}

Le nom de Janus signifie « passage » et le Janus latin est le dieu qui préside à toute espèce de transition d'un état à un autre. Ce passage peut se faire sur trois dimensions : l'espace, le temps et l'être.

Cette figure mythologique de Janus tridimensionnelle est intéressante pour symboliser la place de l'argent. En effet, de même que Janus, l'argent, avec ses fonctions dans les échanges, est un point de passage de l'intérieur vers un extérieur social et inversement (exclusion, insertion sociale). Le temps est consubstantiel du désir ou de sa réalisation, et l'argent vient représenter cette temporalité (futur et passé ou actif et passif). Du point de vue de l'existence enfin, le gardien de la naissance de toute chose que représente Janus trouve un écho dans les fonctions psychosociales de l'argent qui font qu'un individu est ou n'est pas dans notre modernité parce qu'il possède ou non de l'argent.

Si cette figure est intéressante pour penser l'argent de façon générale, elle l'est aussi pour le cas particulier des pratiques tutélaires. Elle synthétise les enjeux liés à l'argent dans ces dispositifs de protection. En effet, en intervenant sur l'argent des personnes suivies, sur ce représentant du passage (d'un extérieur vers un intérieur social et inversement) et en le contrôlant, les pratiques tutélaires héritent de sa problématique : dans quel sens le passage se fait-il pour les personnes protégées ? Si le nombre de mesures levées, comparé à celui des mesures ouvertes, peut concrétiser le passage d'un état à un autre, il faut s'interroger sur le type de passage que constituent effectivement les dispositifs tutélaires. 